\title{
A amostragem da cana-de-açúcar para determinações tecnológicas
}

F. PIMENTEL GOMES, O. VALSECHI, CLOVIS P. DE ABREU e ENIO ROQUE DE OLIVEIRA

Escola Superior de Agricultura \&Luiz de Queiroz»

* Trabalho realizado com o auxílio da Fundação de Amparo à Pesquisa do Estado de São Paulo e da Fundação Rockefeller. 


\section{INTRODUÇÃO}

Nos ensaios com cana-de-açúcar a produção só pode ser corretamente avaliada se, ao lado da quantidade de cana produzida, tivermos dados tecnológicos sôbre o seu valor como matéria prima para as indústrias do açúcar e do álcool. Para consegui-los, é costume colhêr uma amostra de cada parcela, para anảlises tecnológicas.

No Estado de São Paulo há dois métodos principais de amostragem em uso. Um dêles, que chamaremos método A, toma por base a touceira de cana, buscando colhêr uma touceira de cada parcela (CATANI et al., 1959) ou, mais geralmente, 20 canas no sulco, em seguida, em ponto da parcela escolhido ao acaso (ALVAREZ e FREIRE, 1962), método aplicado por técnicos do Instituto Agronômico de Campinas. Na Escola Superior de Agricultura "Luiz de Queiroz", porém, a tendência geral há muitos anos tem sido a de tirar 20 a 30 canas, escolhidas ao acaso na parcela, mas bem distribuídas por tôda a área (ALMEIDA et al., 1952 COURY et al., 1957). No caso de uma parcela com 4 sulcos úteis de 10 metros, por exemplo, tiram-se 5 canas de cada sulco, sendo apenas uma cana de cada ponto sorteado no sulco. Esta técnica será designada método $\mathrm{B}$.

$O$ método $A$, de colheita de uma touceira ou de 20 canas seguidas num ponto do sulco escolhido ao acaso, parte da hipótese de que as maiores diferenças se observam entre colmos distintos, de idades diferentes, da mesma touceira; o método $\mathrm{B}$, de colheita de 20 canas distribuidas por tôda a parcela, admite que também são importantes as diferenças entre touceiras, inclusive as devidas à variação do solo dentro da parcela. Para dar uma base científica sólida à qualquer dos métodos, fazia-se necessário conhecer a variação dentro das touceiras e entre touceiras. Para obter êste conhecimento é que foi realizado o trabalho ora apresentado.

\section{MATERIAL E MÉTODOS}

Foram utilizados 3 talhões industriais de cana-planta, das variedades Co 419, CB 40-69 e CB 41-58, cultivadas em solos de aparência uniforme e localizados na Fazenda Taquaral, no municipio de Piracicaba.

Em cada talhạo demarcaram-se 15 sulcos seguidos e neles tomaram-se 2 blocos (A e B) cada um com todos os sulcos, na 
extensão de $15 \mathrm{~m}$ de comprimento e separados por $5 \mathrm{~m}$ de distância, como indica a Figura 1.

Em cada sulco de cada bloco se escolheu, ao acaso, uma touceira bem caracterizada. Foram, portanto, colhidas 15 touceiras completas de cada bloco, que, depois de despalhadas e numeradas (1 A, $2 \mathrm{~A}, \ldots, 15 \mathrm{~A} ; 1 \mathrm{~B}, 2 \mathrm{~B}, \ldots, 15 \mathrm{~B}$ ), foram pesadas.

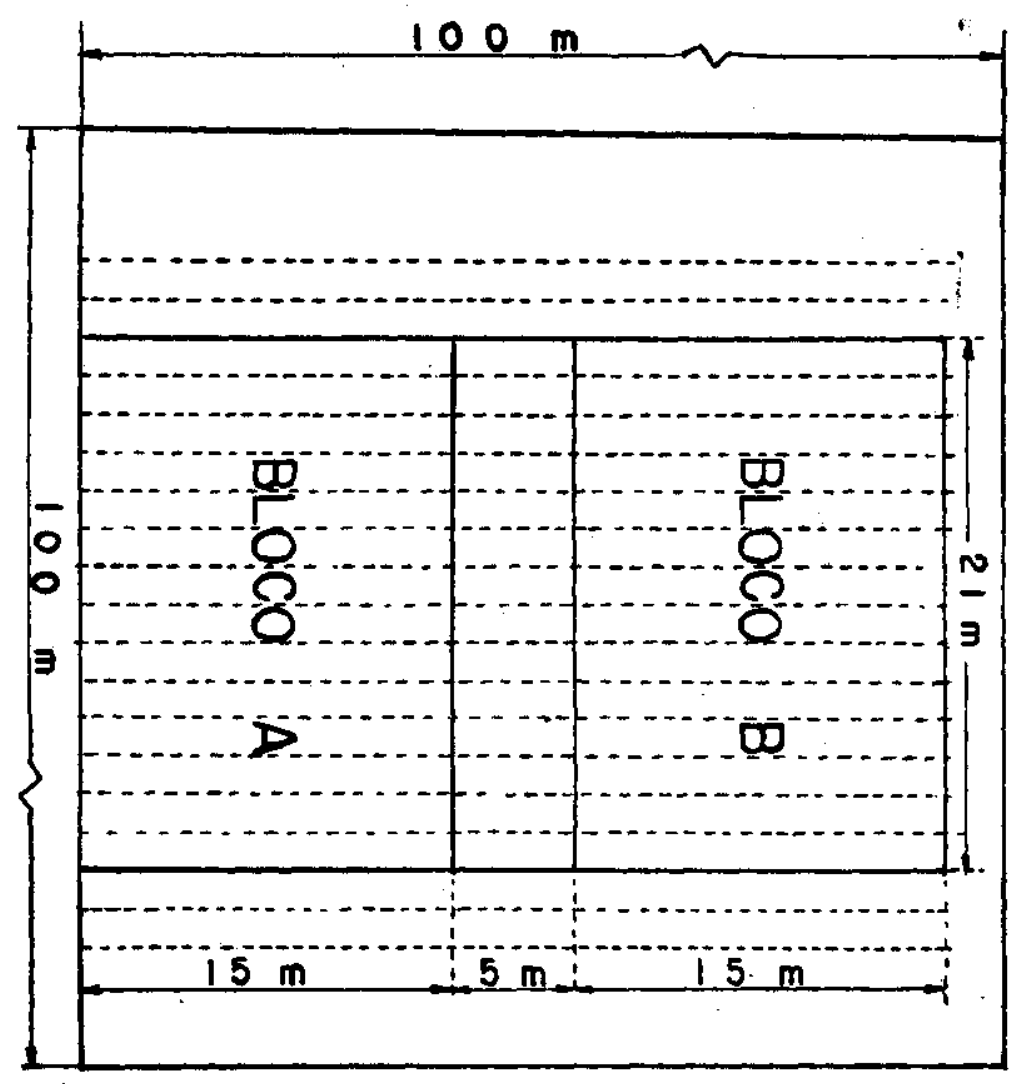

Fig. 1 - Blocos A e B, mostrando os sulcos de cana com as dimensẽes das partes utilizadas.

De cada touceira numerada, foram sorteados 4 colmos. Tinham-se, pois, para cada variedade, 30 touceiras com 4 colmos por touceira, num total de 120 colmos que, em laboratório, foram analisados separadamente, tendo em vista as seguintes determinações.

a) Pêso. Em balança com sensibilidade até $10 \mathrm{~g}$. 
b) Brix. Utilizando-se o hidrômetro para Brix (A. O. A. C., $1960)$.

c) Pol do caldo. Pelo método de Schmitz (BROWNE e ZERBAN, 1941), precipitando-se os sais de cálcio e de chumbo, pela adição da mistura, em partes iguais, de oxalato de potássio a $3 \%$ e fosfato bissódico a $7 \%$.

d) Coeficiente de pureza do caldo. Calculado pela fórmula: (SPENCER e MEADE, 1932).

Coeficiente de pureza $=\frac{\text { Pol x } 100}{\text { Brix }}$

e) Açúcar provável por cento de cana. Dado pela fórmula:

Açúcar provável $\%$ cana $=0,8 \mathrm{Pol}\left(1,4-\frac{40}{\mathrm{C}}\right)$,

de Winter-Carp, na qual $C$, representa o coeficiente de pureza (SPENCER e MEADE, 1932). O fator 0,8 foi usado como uma correção global da extração e da eficiência .

Consideramos, para cada variedade, o modêlo matemático:

$$
y_{i j k}=m+b_{i}+t_{i j}+e_{i j k} \text {, }
$$

onde $m$ é a média da variedade, $b_{i}$ é o efeito do bloco (fixo), $t_{i j}$ é o efeito (aleatório) da touceira $j$ no bloco $i$, e e $e_{i j k}$ é o efeito aleatório da cana $k$ na touceira $j$ do bloco $i$. Temos evidentemente:

$$
\mathrm{i}=1,2 ; \mathrm{j}=1,2, \ldots, 15 ; \mathrm{k}=1,2,3,4 \text {. }
$$

A análise de variância para cada variedade obedece, pois, ao esquema seguinte:

\begin{tabular}{lccc}
\multicolumn{1}{c}{ Causa de variação } & G. L. & Q. M. & E (Q. M.) \\
\hline Blocos & 1 & $Q_{1}$ & $V_{1}=\sigma^{2}+4 \sigma^{2}$ \\
Touceiras d. de blocos & 28 & $Q_{2}$ & $V_{2}=\sigma^{2}$ \\
\hline
\end{tabular}

Neste quadro, Q. M. indica Quadrado Médio, e E (Q. M.) é a esperança natemática correspondente. de $t_{i j}$.

Por outro lado, $\sigma^{2}$ é a variância de $\mathrm{e}_{\mathrm{ijk}}$ e $\sigma^{2}{ }_{1}$ é a variância

Conclui-se, pois, que $\sigma^{2}$ é estimado por:

$\mathrm{s}^{2}=\mathrm{Q}_{2}=\mathrm{Q}$. M. Canas dentro das touceiras e que $\boldsymbol{\sigma}^{2}{ }_{1}$ é estimado por 


$$
\begin{gathered}
\mathrm{s}_{1}{ }_{1}=\frac{1}{4}\left(\mathrm{Q}_{1}-\mathrm{Q}_{2}\right) \\
=\frac{1}{4}\left[\begin{array}{c}
\mathrm{Q} \cdot \mathrm{M} . \\
-\mathrm{Q} . \mathrm{M} \text {. Touceiras dentro dos blocos }-
\end{array}\right]
\end{gathered}
$$

Considerando-se, para exemplificar, uma amostra de 20 canas, no caso de 20 canas de mesma touceira (método $\mathrm{A}$ ), a variância da média seria:

$\mathrm{V}\left(m_{2 \mathrm{o}}\right)=\frac{\sigma^{2}+20 \sigma^{2}{ }_{1}}{20}=\frac{\sigma^{2}}{20}+\sigma_{1}^{2}=\frac{5 \mathrm{~V}_{1}-4 \cdot \mathrm{V}_{2}}{20}$,

ao passo que com 20 canas, mas uma de cada touceíra (método B), obteríamos:

$$
\mathrm{V}\left(m_{1}\right)=\frac{\sigma^{2}+\sigma^{2}{ }_{1}}{20}=\frac{1}{20} \cdot \frac{V_{1}+3 V_{2}}{4} .
$$

Admitindo êste modêlo matemático, devemos ter sempre $\mathrm{V}_{1}>\mathrm{V}_{2}$ e resulta ainda que

$$
\mathrm{V}\left(m_{20}\right)<\left(m_{1}\right)
$$

em qualquer caso, o que dá invariàvelmente menor variância à amostra obtida com uma só cana por touceira.

No entanto, há a possibilidade de têrmos $V_{1}<V_{2}$, como sugerem COCHRAN e COX (1957). Com efeito, podemos considerar o modêlo

$$
y_{i j k}=m+b_{i}+e_{i j k}
$$

admitindo que para duas canas da mesma touceira $j$ temos um coeficiente de correlação $\rho$. Nestas condições a análise de variância nos dá as esperanças matemáticas expostas a seguir.
Causa de variação
G. L.
Q. M.
E (Q. M.)

Blocos

Touceiras d. de blocos

Canas d. de touceiras

$\begin{array}{lll}1 & Q_{1} & V_{1}=\sigma^{2}(1+3 \rho) \\ 28 & Q_{2} & V_{2}=\sigma^{2}(1-\rho)\end{array}$

No caso de têrmos $\rho>0$, então $V_{1}>V_{2}$ e recaímos no caso anterior. Mas se tivermos $\rho<0$, então fica $V_{1}<V_{2}$, caso que $m=$ estimativa da média. 
não podíamos antes admitir. Êste modêlo é, pois, mais geral.

É fácil verificar que temos:

$$
\begin{aligned}
\sigma^{2} & =\frac{V_{1}+3 V_{2}}{4}, \\
\rho & =\frac{V_{1}-V_{2}}{V_{1}+3 V_{2}},
\end{aligned}
$$

de onde resulta que as estimativas respectivas são:

$$
\begin{aligned}
& \mathrm{s}^{2}=\frac{\mathrm{Q}_{\mathrm{I}}+3 \mathrm{Q}_{2}}{4}, \\
& \mathrm{r}=\frac{\mathrm{Q}_{1}-\mathrm{Q}_{2}}{\mathrm{Q}_{1}+3 \mathrm{Q}_{2}} .
\end{aligned}
$$

Nestas condições temos para o caso de 20 cañas por touceira:

$$
\mathrm{V}\left(m_{20}\right)=\frac{\sigma^{2}(1+19 \rho)}{20}=\frac{5 \mathrm{~V}_{1}-4 \mathrm{~V}_{2}}{20} \text {. }
$$

Para o caso de uma cana por touceira fica:

$$
\mathrm{V}\left(m_{1}\right)=\frac{\sigma^{2}}{20}=\frac{1}{20} \frac{\mathrm{V}_{1}+3 \mathrm{~V}_{2}}{4} .
$$

Estes resultađos coincidem com os anteriores no caso de $V_{1} \geq V_{2}$, mas também se mantêm no caso de $V_{1}<V_{2}$, em que temos $\rho<0$. São, pois, mais gerais.

- Podemos, pois, em qualquer caso, adotar as fórmulas seguintes para as variâncias das amostras de 20 canas por touceira $\left(m_{20}\right)$ e de 20 sanas, uma só por touceira $\left(m_{1}\right)$ :

$$
\begin{gathered}
\mathrm{V}\left(m_{20}\right)=\frac{5 \mathrm{~V}_{1}-4 \mathrm{~V}_{2}}{20} \\
\mathrm{~V}\left(m_{1}\right)=\frac{1}{20} \cdot \frac{\mathrm{V}_{1}+3 \mathrm{~V}_{2}}{4} .
\end{gathered}
$$

As estimativas respectivas serão:

$$
\mathrm{V}\left(m_{2 \mathrm{o}}\right)=\frac{5 \mathrm{Q}_{1}-4 \mathrm{Q}_{2}}{20},
$$

$\overline{m=\text { estimativa da média. }}$ 


$$
\mathrm{V}\left(m_{1}\right)=\frac{1}{20} \cdot \frac{\mathrm{Q}_{1}+3 \mathrm{Q}_{2}}{4} .
$$

\section{RESULTADOS E DISCUSSÃO}

Os dados das análises tecnológicas referentes às 3 variedades estudadas constam dos quadros 1,2 e 3 . Como nenhuma escolha foi feita, colhendo-se tôdas as canas de cada touceira, ocorreram alguns colmos de pêso muito baixo.

$\bar{m}=$ estimativa da média. 


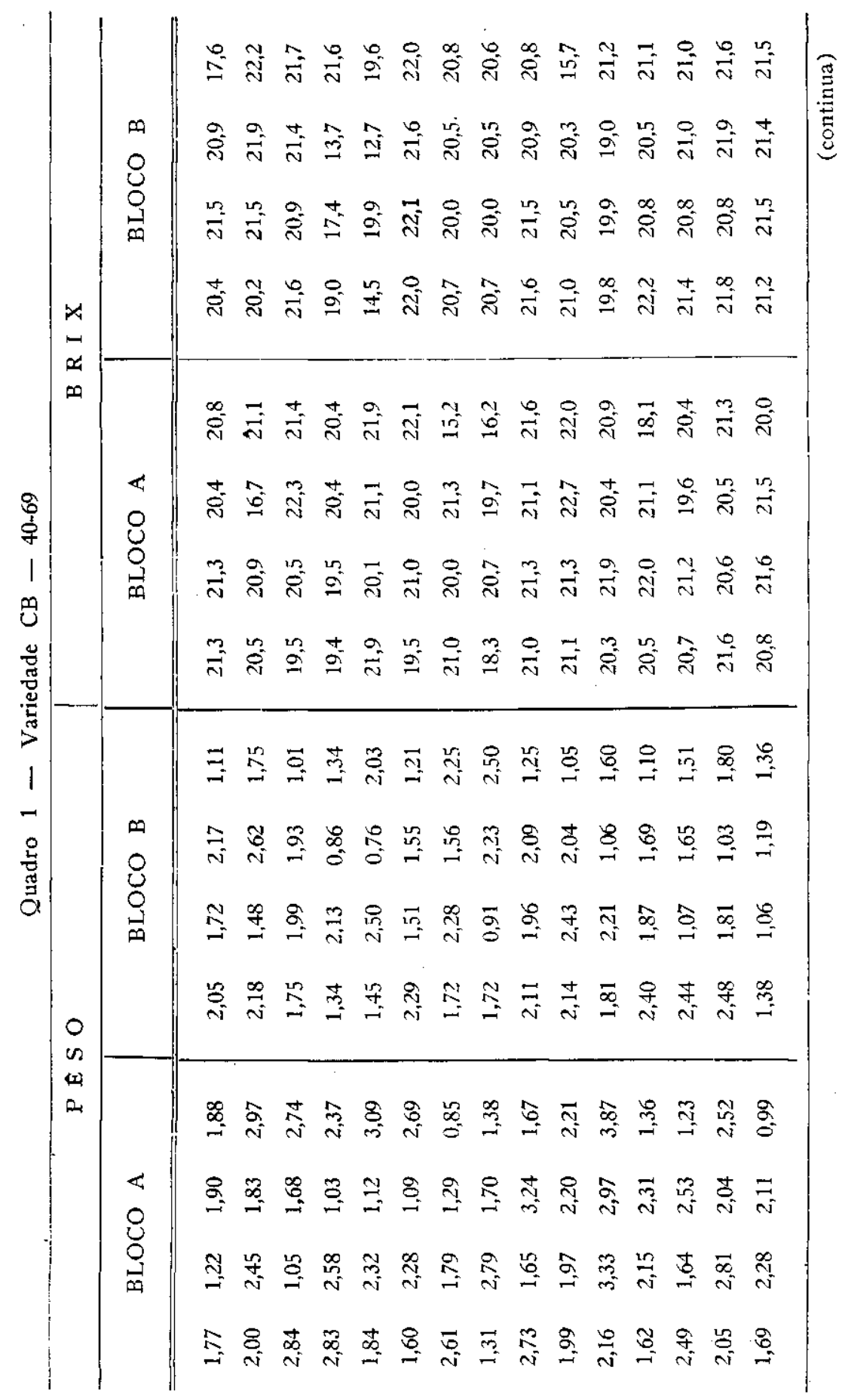




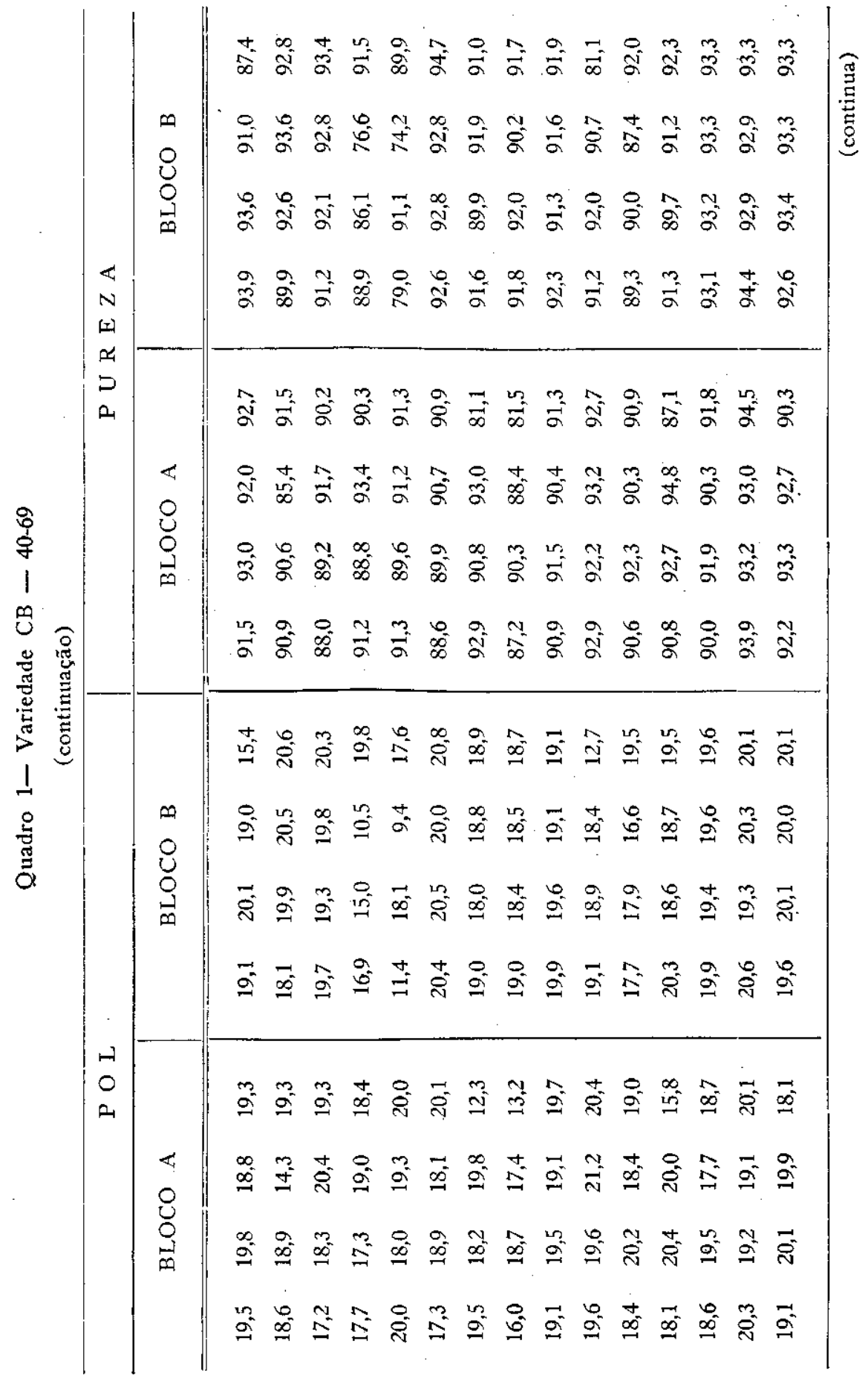


Quadro 1 - Variedade CB - 40-69

(continuação)

\begin{tabular}{|c|c|c|c|c|c|c|c|}
\hline & $B L O$ & $O A$ & & & $\mathrm{BLO}$ & $\mathrm{O} B$ & \\
\hline 15,0 & 15,4 & 14,5 & 14,9 & 14,9 & 15,7 & 14,6 & 11,6 \\
\hline 14,3 & 14,5 & 10,6 & 14,9 & 13,8 & 15,4 & 15,9 & 16,0 \\
\hline 13,0 & 13,9 & 15,8 & 14,8 & 15,8 & 14,9 & 15,4 & 15,7 \\
\hline 13,6 & 13,1 & 14,8 & 14,1 & 12,8 & 11,2 & 7,4 & 15,2 \\
\hline 15,4 & 13,7 & 14,9 & 15,4 & 8,2 & 13,9 & 6,5 & 13,4 \\
\hline 13,1 & 14,4 & 13,9 & 15,4 & 15,8 & 15,9 & 15,5 & 16,3 \\
\hline 15,1 & 13,9 & 15,4 & 8,4 & 14,6 & 13,7 & 14,5 & 14,5 \\
\hline 12,0 & 14,3 & 13,2 & 9,6 & 14,7 & 14,2 & 14,2 & 14,4 \\
\hline 14,6 & 15,0 & 14,6 & 15,2 & 15,4 & 15,1 & 14,8 & 14,8 \\
\hline 15,2 & 15,2 & 16,4 & 15,8 & 14,7 & 14,5 & 14,1 & 9,3 \\
\hline 14,1 & 15,6 & 14,1 & 14,6 & 13,5 & 13,7 & 12,5 & 15,1 \\
\hline 14,3 & 15,8 & 15,6 & 11,9 & 15,6 & 14,2 & 14,4 & 15,1 \\
\hline 14,2 & 15,0 & 13,5 & 14,4 & 15,5 & 15,0 & 15,2 & 15,2 \\
\hline 15,8 & 14,9 & 14,8 & 15,7 & 16,1 & 15,0 & 15,8 & 15,7 \\
\hline 14,8 & 15,7 & 15,4 & 13,8 & 15,2 & 15,6 & 15,5 & 15,6 \\
\hline
\end{tabular}




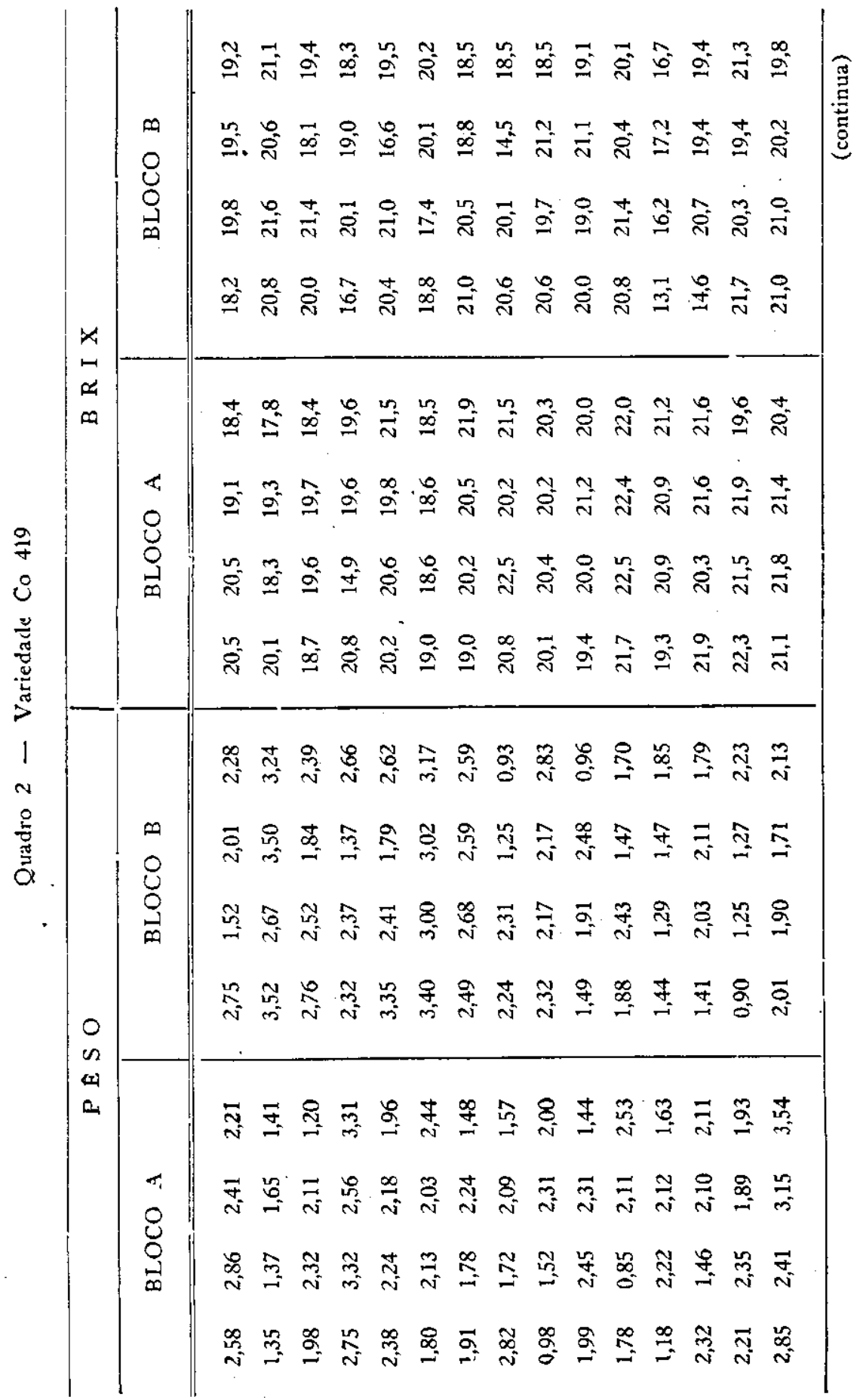




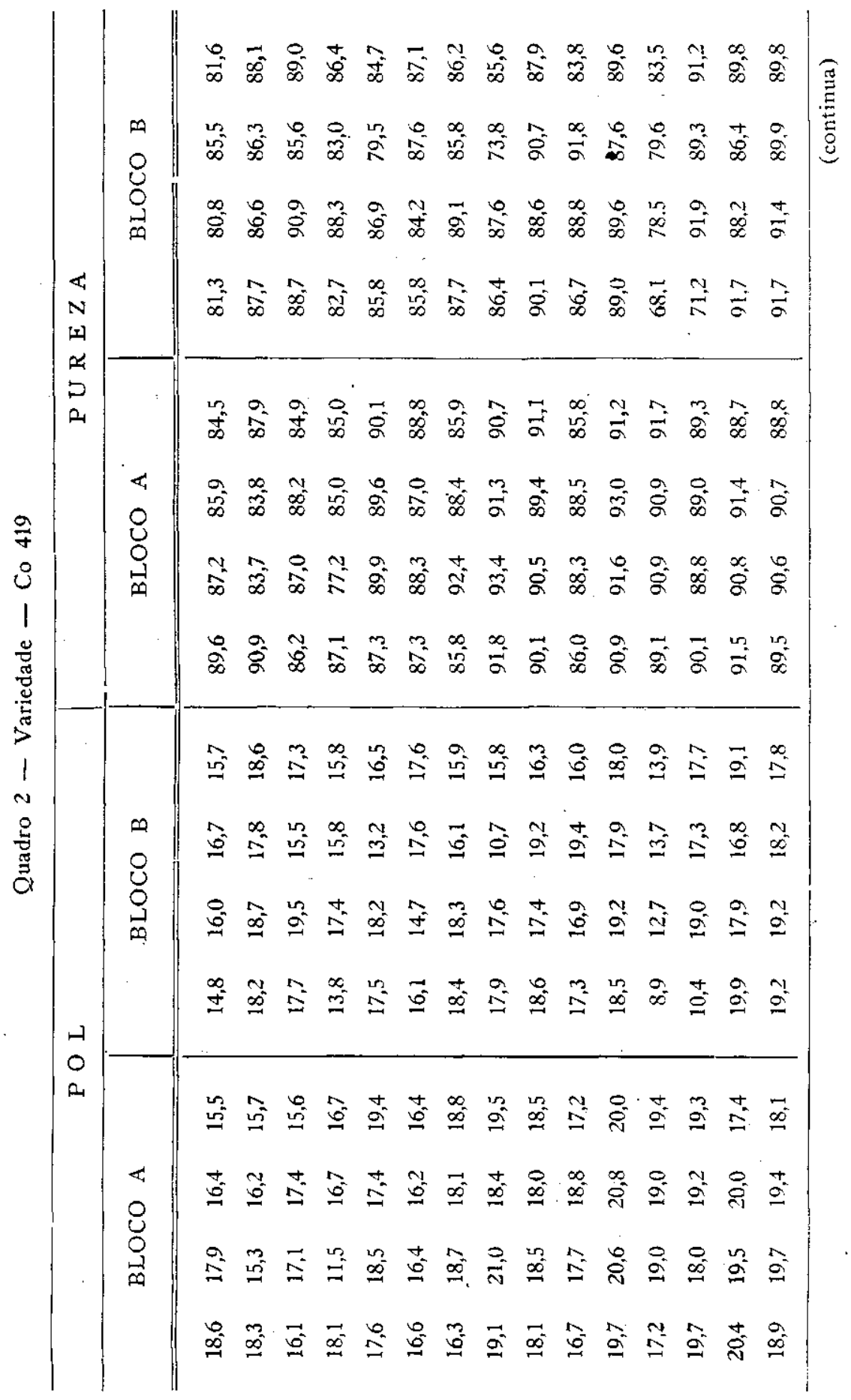




\section{Quadro 2 - Variedade Co 419}

(continuação)

A ÇU C A R P ROVA VE L

\begin{tabular}{rrrl|rrrr}
\hline \multicolumn{3}{c}{ BLOCO A } & \multicolumn{4}{|c}{ BLOCO B } \\
\hline 14,0 & 13,5 & 12,3 & 11,5 & 10,7 & 11,6 & 12,4 & 11,4 \\
14,0 & 11,3 & 11,9 & 11,8 & 13,8 & 14,0 & 13,3 & 14,1 \\
12,1 & 12,8 & 14,1 & 11,6 & 13,5 & 14,9 & 11,6 & 13,1 \\
13,6 & 8,1 & 12,4 & 12,4 & 10,1 & 13,4 & 14,0 & 11,9 \\
13,3 & 14,1 & 13,5 & 14,8 & 13,0 & 13,7 & 9,5 & 11,5 \\
12,5 & 12,4 & 12,2 & 12,5 & 12,1 & 10,8 & 13,3 & 13,2 \\
12,2 & 14,4 & 13,7 & 14,1 & 13,9 & 13,9 & 12,5 & 11,9 \\
14,7 & 16,3 & 14,2 & 15,0 & 13,4 & 13,3 & 7,3 & 11,8 \\
13,8 & 14,1 & 13,8 & 14,3 & 14,2 & 13,2 & 14,8 & 12,3 \\
12,5 & 13,4 & 14,2 & 12,8 & 14,0 & 12,8 & 15,0 & 11,8 \\
15,1 & 15,9 & 16,2 & 15,4 & 14,1 & 14,6 & 13,5 & 13,7 \\
13,1 & 14,5 & 14,6 & 15,0 & 5,8 & 9,0 & 9,8 & 10,3 \\
15,1 & 13,7 & 14,6 & 14,7 & 7,0 & 14,7 & 13,2 & 16,6 \\
15,7 & 15,0 & 15,4 & 14,1 & 15,4 & 13,7 & 12,7 & 14,5 \\
14,4 & 15,1 & 14,9 & 13,8 & 14,8 & 14,8 & 13,9 & 13,6 \\
\hline
\end{tabular}




\begin{tabular}{|c|c|c|}
\hline & & 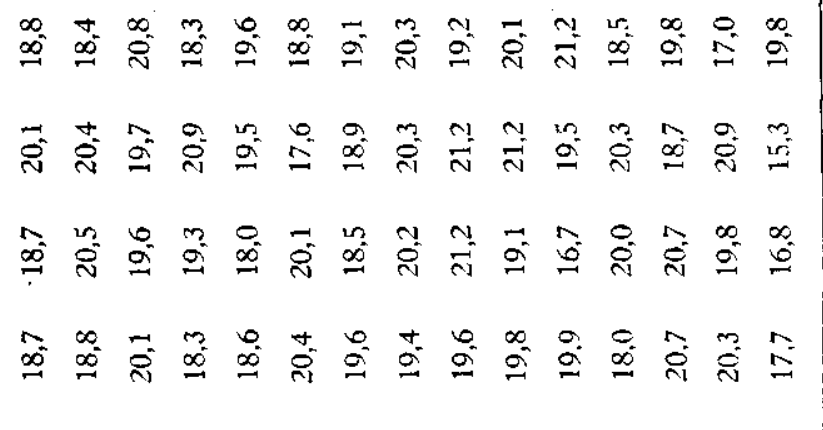 \\
\hline$\oplus$ & & 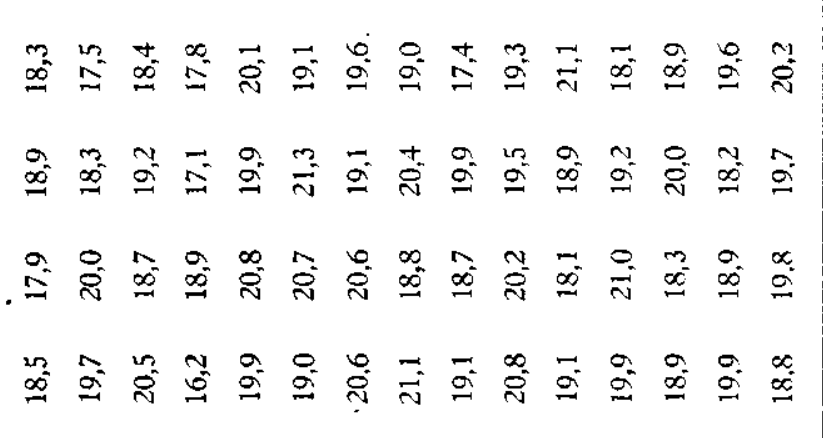 \\
\hline $\begin{array}{l}0 \\
0\end{array}$ & & 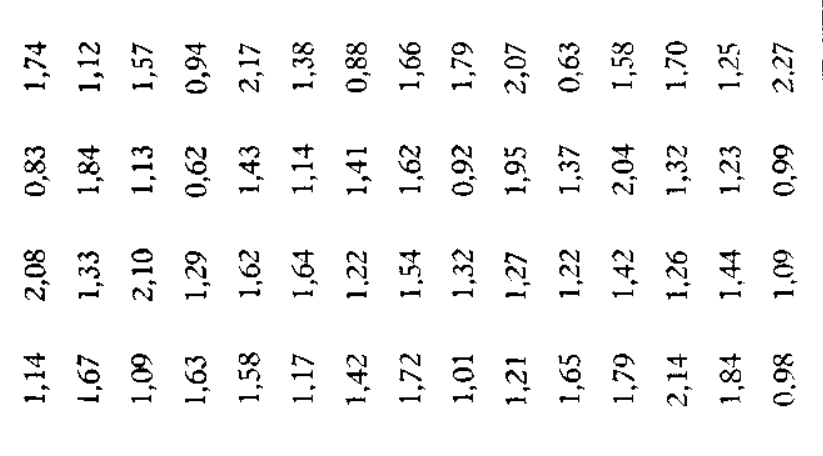 \\
\hline $\begin{array}{l}0 \\
0 \\
2\end{array}$ & $\begin{array}{l}\varangle \\
\stackrel{U}{0} \\
\stackrel{\Delta}{0}\end{array}$ & 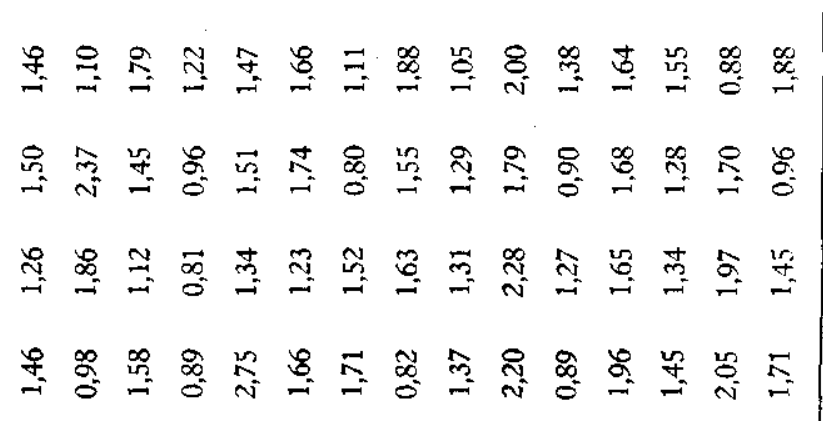 \\
\hline
\end{tabular}




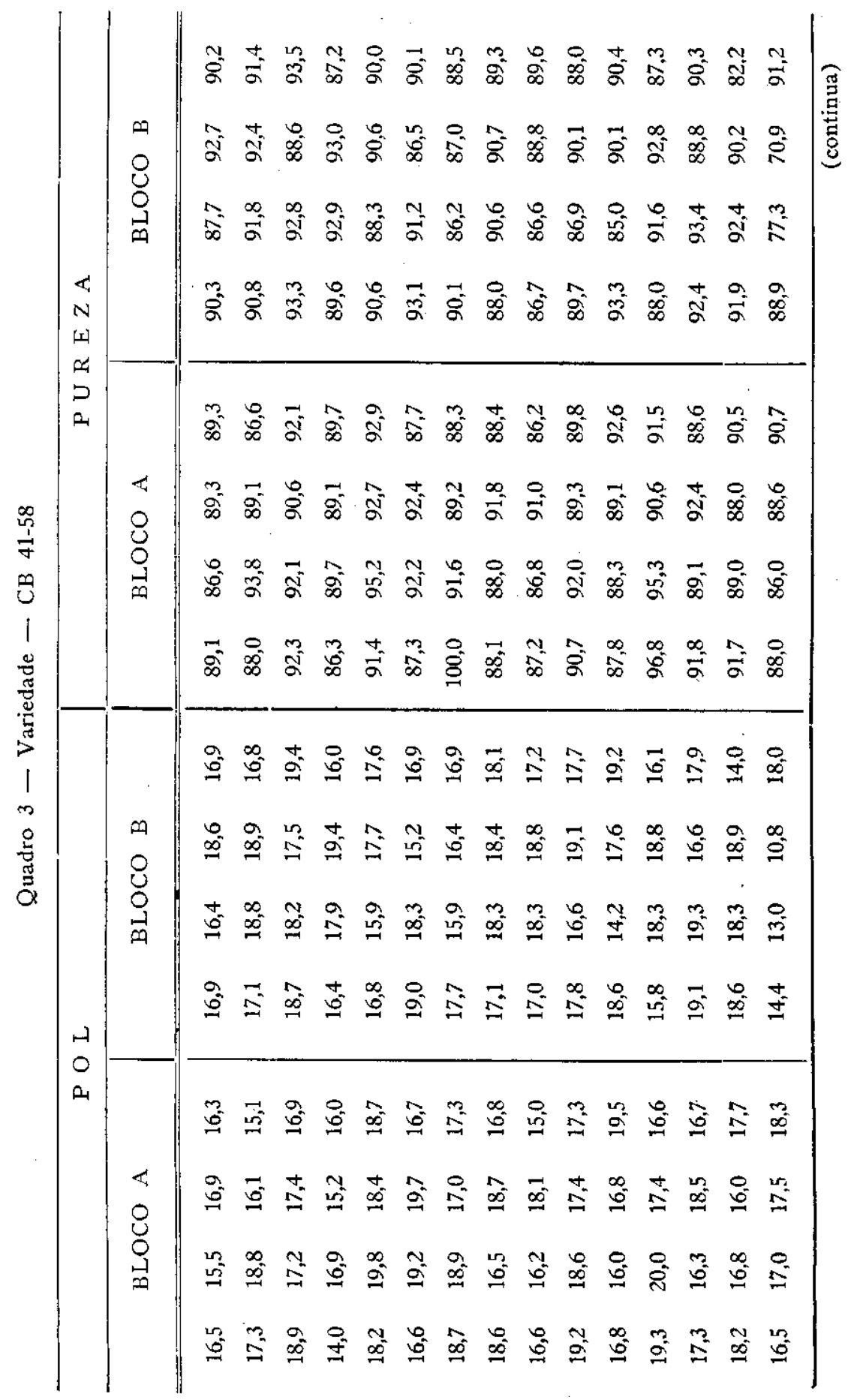


Quadro 3 - Variedade - CB 41-58

(continuação)

A ÇUCAR P R O A A E L

\begin{tabular}{cccc|rrrr}
\hline \multicolumn{3}{c}{ BLOCO A } & & \multicolumn{3}{|c}{ BLOCO B } \\
\hline \hline 12,5 & 11,6 & 12,9 & 12,4 & 12,9 & 12,4 & 14,4 & 13,0 \\
13,1 & 14,6 & 12,2 & 11,4 & 13,1 & 14,5 & 14,6 & 12,9 \\
14,6 & 13,3 & 14,3 & 13,1 & 14,6 & 14,1 & 13,2 & 15,1 \\
10,5 & 12,9 & 11,6 & 12,2 & 12,5 & 13,9 & 15,1 & 12,0 \\
14,0 & 15,5 & 14,3 & 14,5 & 12,9 & 12,0 & 13,5 & 13,5 \\
13,2 & 14,8 & 15,2 & 12,6 & 14,7 & 14,0 & 11,4 & 13,0 \\
14,4 & 14,5 & 13,0 & 13,1 & 13,5 & 11,9 & 12,4 & 12,8 \\
14,1 & 12,6 & 14,4 & 12,7 & 12,9 & 14,0 & 14,3 & 13,8 \\
12,5 & 12,2 & 13,9 & 11,2 & 12,8 & 13,8 & 14,3 & 13,1 \\
14,9 & 14,3 & 13,3 & 13,2 & 13,6 & 12,5 & 14,6 & 13,4 \\
12,7 & 12,1 & 12,8 & 15,1 & 14,4 & 10,5 & 13,4 & 14,7 \\
15,2 & 15,7 & 14,3 & 12,8 & 12,0 & 14,1 & 14,6 & 12,1 \\
14,3 & 12,4 & 14,3 & 12,7 & 14,8 & 15,0 & 12,6 & 13,7 \\
14,1 & 12,8 & 12,2 & 13,6 & 14,4 & 14,1 & 14,4 & 10,2 \\
12,5 & 12,7 & 13,2 & 14,1 & 10,5 & 9,2 & 7,3 & 13,9 \\
\hline
\end{tabular}




\subsection{Variedade $C B 40.69$}

Brix

A análise de variância deu os resultados seguintes.
Causa de variaçĩo
G. L.
S. Q.
Q. M.

\begin{tabular}{lccc}
\hline Blocos & 1 & 0,70 & 0,70 \\
Touceiras dentro de blocos & 28 & 154,47 & $5,52\left(Q_{1}\right)$ \\
Canas dentro de touceiras & 90 & 184,77 & $2,05\left(Q_{2}\right)$ \\
\hline
\end{tabular}

Para uma amostra de 20 canas, de uma só touceira, a estimativa da variância de seu Brix seria, pois,

$$
V\left(m_{20}\right)=\frac{5 \mathrm{Q}_{1}-4 \mathrm{Q}_{2}}{20}=0,972,
$$

ao passo que uma amostra de 20 canas, mas com apenas uma cana por touceira, nos daria:

$$
V\left(m_{1}\right)=\frac{1}{20} \frac{\mathrm{Q}_{1}+3 \mathrm{Q}_{2}}{4}=0,146 .
$$

Como a média geral estimada foi $m=20,48$, verifica-se que no caso de 20 canas de uma só touceira, o coeficiente de variação seria de $4,8 \%$, ao passo que com uma só cana por touceira êle cairia para $1,9 \%$.

Pol

A análise estatística seguiu marcha análoga, com os resultados dados a seguir.
Causa de variação
G. L.
S. Q.
Q.M.

$\begin{array}{lrcc}\text { Blocos } & 1 & 0,3255 & 0,33 \\ \text { Touceiras dentro de blocos } & 28 & 228,5251\left(Q_{1}\right) & 8,16 \\ \text { Canas dentro de touceiras } & 90 & 265,1727\left(Q_{2}\right) & 2,95\end{array}$

A média geral é $m=18,66$.

$V=$ estimativa da variância.

$m=$ estimativa da média. 
Temos agora

$$
\begin{aligned}
& V\left(m_{20}\right)=\frac{5 \mathrm{Q}_{1}-4 \mathrm{Q}_{2}}{20}=1,45, \text { C. } \mathrm{V} .=6,4 \%, \\
& V\left(m_{1}\right)=\frac{1}{20} \frac{\mathrm{Q}_{1}+3 \mathrm{Q}_{2}}{4}=0,21, \text { C. } \mathrm{V} .=2,5 \% .
\end{aligned}
$$

\section{Pureza}

Causa de variação

G. L.

S. Q.

Q. M.

\section{Blocos}

Touceiras dentro de blocos

Canas dentro de touceiras

$\begin{array}{rrr}1 & 0,0380 & 0,038 \\ 28 & 602,0370 & 21,50 \\ 90 & 707,3653 & 7,86\end{array}$

A média geral é $m=90,83$.

Temos pois,

$$
\begin{array}{ll}
V\left(m_{20}\right)=3,80 \\
V\left(m_{1}\right)=0,56
\end{array} \quad, \quad \begin{array}{ll}
\text { C. V. }=2,1 \% \\
\text { C. V. }=0,83 \% .
\end{array}
$$

Açúcar Provável

\begin{tabular}{lrrr}
\multicolumn{1}{c}{ Causa de variação } & G. L. & S. Q. & Q. M. \\
\hline Blocos & 1 & 0,0907 & 0,091 \\
Touceiras dentro de blocos & 28 & 169,7822 & 6,06 \\
Canas dentro de touceiras & 90 & 193,3471 & 2,15 \\
\hline
\end{tabular}

A média geral é $m=14,35$.

Temos agora:

$$
\begin{array}{lll}
V\left(m_{20}\right)=1,09 & , & \text { C. } V .=7,3 \% \\
V\left(m_{1}\right)=0,156 & \text { C. V. }=2,7 \% .
\end{array}
$$

Pêso

\begin{tabular}{lrrr} 
Causa de variação & G. L. & S. Q. & Q. M. \\
\hline Blocos & 1 & 3,7489 & 3,7489 \\
Touceiras dentro de blocos & 28 & 9,4677 & 0,3381 \\
Canas dentro de touceiras & 90 & 30,9764 & 0,3442 \\
\hline
\end{tabular}

$V=$ estimativa da variância.

$m=$ estimativa.da média. 
A média geral é $m=1,90 \mathrm{~kg}$.

No caso presente obtemos:

$$
\begin{aligned}
& V\left(m_{20}\right)=0,0157 \quad, \quad \text { C. V. }=6,57 \% \text {, } \\
& V\left(m_{1}\right)=0,0171 \quad, \quad \text { C. } V .=6,89 \% \text {. }
\end{aligned}
$$

Para os caracteres estudados há, pois, no caso da variedade $C B$ 40-69, considerável redução na varịância e no coeficiente de variação, quando tomamos uma só cana por touceira. Há, porém, uma exceção, relativa ao pêso das canas, em que há uma ligeira vantagem a favor do uso de amostras de 20 canas de uma só touceira.

\subsection{Variedade Co 419}

Brix

\begin{tabular}{lrrr}
\multicolumn{1}{c}{ Causa da variação } & G. L. & S. Q. & Q. M. \\
\hline Blocos & 1 & & 22,88 \\
Touceiras dentro de blocos & 28 & 159,32 & 5,69 \\
Canas dentro de touceiras & 90 & 150,37 & 1,67 \\
\hline
\end{tabular}

A média geral é $m=19,84$.

Temos, pelos resultados da análise de variância:

$$
V\left(m_{1}\right)=0,13 \quad \text {, } \quad \text { C. V. }=1,8 \% \text {. }
$$

Pol

\begin{tabular}{lrrr}
\multicolumn{1}{c}{ Causa da variação } & G. L. & S. Q. & Q. M. \\
\hline Blocos & 1 & 43,5969 & 43,60 \\
Touceiras dentro de blocos & 28 & 253,0672 & 9,04 \\
Canas dentro de touceiras & 90 & 220,7649 & 2,45
\end{tabular}

A média geral é $\dot{m}=17,41$.

Temos no caso presente:

$$
\begin{aligned}
& V\left(m_{20}\right)=1,77 \quad, \quad \text { C. } V .=7,6 \%, \\
& V\left(m_{1}\right)=0,21 \quad, \quad \text { C. V. }=2,6 \% \text { ． }
\end{aligned}
$$

$V=$ estimativa da variância.

$m=$ estimativa da média. 


\section{Pureza}

\begin{tabular}{lccc} 
Causa de variação & G. L. & S.Q. & Q. M. \\
\hline Blocos & 1 & 181,1711 & 181,17 \\
Tonceiras dentro de blocos & 28 & 933,2286 & 33,33 \\
Canas dentro de touceiras & 90 & 844,4429 & 9,83 \\
\hline
\end{tabular}

A média é $m=87,45$.

As estimativas das variâncias são:
$V\left(m_{20}\right)=6,37$
C. V. $=2,9 \%$,
$V:\left(m_{1}\right)=0,79$
C. V. $=1,0 \%$.

Açúcar Provável

\begin{tabular}{lccc} 
Causa de variação & G. L. & S. Q. & Q. M. \\
\hline Blocos & 1 & 21,9071 & 21,91 \\
Tonceiras dentro de blocos & 28 & 191,0330 & 6,82 \\
Canas dentro de touceiras & 90 & 182,9166 & 2,03
\end{tabular}

A média geral é $m=13,21$.

As estimativas das variâncias são:
$V\left(m_{2 o}\right)=1,30$
$V\left(m_{1}\right)=0,16$
C. V. $=8,6 \%$,
C. V. $=3,0 \%$.

Pêso

\begin{tabular}{lrrr}
\multicolumn{1}{c}{ Causa de variação } & G. L. & S. Q. & Q. M. \\
\hline Blocos & 1 & 0,1527 & 0,1527 \\
Touceiras dentro de blocos & 28 & 27,2650 & 0,9737 \\
Canas dentro de touceiras & 90 & 17,1300 & 0,1903
\end{tabular}

A média geral é $m=2,13$.

Temos agora:

$$
\begin{aligned}
& V\left(m_{20}\right)=0,2053 \quad, \quad \text { C. V. }=21,2 \%, \\
& V \cdot\left(m_{1}\right)=0,0193 \quad, \quad \text { C. V. }=6,5 \% \text {. }
\end{aligned}
$$

\subsection{Variedade $C B \quad 41.58$}

$V=$ estimativa da variância.

$m=$ estimativa da média. 
Brix

\begin{tabular}{lcrc}
\multicolumn{1}{c}{ Causa de variação' } & G. L. & S. Q. & Q. M. \\
\hline Blocos & 1 & 0,28 & 0,28 \\
Touceiras dentro de blocos & 28 & 57,38 & 2,05 \\
Canas dentro de touceiras & 90 & 99,98 & 1,11 \\
\hline
\end{tabular}

A média geral é $m=19,34$.

Temos, pelos resultados da análise da variância:

$$
\begin{array}{lll}
V\left(m_{20}\right)=0,29 & , & \text { C. V. }=2,8 \% \\
V\left(m_{1}\right)=0,07 & \text { C. V. }=1,4 \% .
\end{array}
$$

Pol

\begin{tabular}{lrrr} 
Causa de variação & G. .. & S.Q. & Q.M. \\
\hline Blocos & 1 & 0,0472 & 0,0472 \\
Touceiras dentro de blocos & 28 & 97,7237 & 3,4901 \\
Canas dentro de touceiras & 90 & 166,2386 & 1,8471
\end{tabular}

A média geral é $m=17,36$.

As estimativas das variâncias são:

$$
\begin{array}{ll}
V\left(m_{20}\right)=0,5030 \\
V\left(m_{1}\right)=0,1129
\end{array} \quad, \quad \begin{aligned}
& \text { C. } V .=4,1 \% \\
& \text { C. } V .=1,9 \%
\end{aligned} \text {. }
$$

Pureza

\begin{tabular}{lrrr}
\multicolumn{1}{c}{ Causa de variação } & G. L. & S. Q. & Q. M. \\
\hline Blocos & 1 & 12,1470 & 12,1470 \\
Touceiras dentro de blocos & 28 & 469,2200 & 16,7578 \\
Canas dentro de touceiras & 90 & 710,7590 & 7,8973
\end{tabular}

A média geral é $m=89,71$.

As estimativas das variâncias são:

$$
\begin{aligned}
& V\left(m_{20}\right)=2,6100 \quad, \quad \text { C. } V .=1,8 \% \text {, } \\
& V\left(m_{1}\right)=0,5056 \quad, \quad \text { C. V. }=0,8 \% \text {. }
\end{aligned}
$$

$V=$ estimativa da variância.

$m=$ estimativa da média. 
Açúcar Provável

\begin{tabular}{lrrr} 
Causa de variação & G. L. & S.Q. & Q. M. \\
\hline Blocos & 1 & 0,5741 & 0,5741 \\
Touceiras dentro de blocos & 28 & 81,8279 & 2,9224 \\
Canas dentro de touceiras & 90 & 127,2490 & 1,4139 \\
\hline
\end{tabular}

A média geral é $m=13,29$.

As estimativas das variâncias são:

$$
\begin{array}{lll}
V\left(m_{20}\right)=0,4478 & , & \text { C. } V .=5,0 \% \\
V\left(m_{1}\right)=0,0895 & \text { C. V. }=2,2 \% .
\end{array}
$$

Pêso

\begin{tabular}{lrrr}
\multicolumn{1}{c}{ Causa de variação } & G. L. & S. Q. & Q. M. \\
\hline Blocos & 1 & 0,0563 & 0,0563 \\
Touceiras dentro de blocos & 28 & 6,1053 & 0,2180 \\
Canas dentro de touceiras & 90 & 13,0464 & 0,1450 \\
\hline
\end{tabular}

A média geral é $m=1,46$.

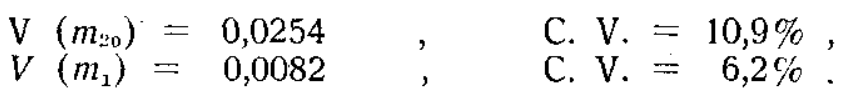

\section{DISCUSSÃO DOS RESULTADOS}

Para as três variedades estudadas e para todos os dados de Brix, pol, pureza, açúcar provável por cento de cana e pêso há vantagem no uso de uma só cana por touceira, em lugar de 10 ou 20 , pois, para um mesmo número de canas, a precisão máxima é obtida quando é máximo o número de touceiras, isto é, quando se toma só uma cana por touceira.

A única exceção ocorreu no caso do pêso, na variedade CB 40-69, em que houve resultado levemente favorável ao uso de mais canas por touceira.

O quadro seguinte, que dá os coeficientes de variação obtidos para os diversos casos, demonstra bem o que acabamos de dizer.

$V=$ estimativa da variância .

$m=$ estimativa da média. 
Coeficiente de variação de amostras de 20 canas por parcela.

\begin{tabular}{|c|c|c|c|}
\hline Variedade & Característica & Com 20 canas por & $\begin{array}{l}\text { Com } 1 \text { cana por } \\
\text { touceira }\end{array}$ \\
\hline CB $40-69$ & $\begin{array}{l}\text { Brix } \\
\text { Pol } \\
\text { Pureza } \\
\text { Acúcar provável } \\
\text { Pêso }\end{array}$ & $\begin{array}{l}4,8 \% \\
6.4 \% \\
2,1 \% \\
7.3 \% \\
6,6 \%\end{array}$ & $\begin{array}{l}1,9 \% \\
2,5 \% \\
0,83 \% \\
2,7 \% \\
6,9 \%\end{array}$ \\
\hline Co 419 & $\begin{array}{l}\text { Brix } \\
\text { Pol } \\
\text { Pureza } \\
\text { Açúcar provável } \\
\text { Péso }\end{array}$ & $\begin{array}{r}5.3 \% \\
7,6 \% \\
2.9 \% \\
8,6 \% \\
21.2 \%\end{array}$ & $\begin{array}{l}1,8 \% \\
2,6 \% \\
1,0 \% \\
3,0 \% \\
6,5 \%\end{array}$ \\
\hline CB 41-58 & $\begin{array}{l}\text { Brix } \\
\text { Pol } \\
\text { Pureza } \\
\text { Açúcar provável } \\
\text { Pćso }\end{array}$ & $\begin{array}{r}2,8 \% \\
4,1 \% \\
1,8 \% \\
5,0 \% \\
10,9 \%\end{array}$ & $\begin{array}{l}1,4 \% \\
1,9 \% \\
0,8 \% \\
2,2 \% \\
6,2 \%\end{array}$ \\
\hline
\end{tabular}

Para o açúcar provável, que é talvez a mais importante das caracteristicas estudadas, o coeficiente de variação médio, para as três variedades e com uma só cana por touceira (método B), foi de $2,7 \%$. Admitido êste valor, um ensaio com 5 tratamentos e 6 repetições, por exemplo; do qual obtivéssemos de cada parcela uma amostra de 20 canas, tiradas uma de cada touceira, teríamos uma diferença mínima significativa, ao nivel de $5 \%$ de probabilidade, de $4,7 \%$ de açúcar provável, pelo teste de Tukey (ver PIMENTEL GOMES, 1963) e 3,3\% pelo teste de $t$. Para o caso do método A modificado (20 canas seguidas em um ponto escolhido ao acaso) o coeficiente de variação médio seria de $7,0 \%$ e as diferenças mínimas significativas, em condições análogas às referidas acima, seriam de $15,1 \%$ para o caso do teste de Tukey e de $12,1 \%$ para 0 teste $t$.

Ora, as diferenças de composição da cana por efeito de adubação raramente vão além de 3 ou $4 \%$ do teor médio de açúcar (PIMENTEL GOMES e CARDOSO, 1958), isto é, de 4 a $5 \mathrm{~kg}$ de açúcar por tonelada de cana. Segue-se, pois, que o método $\mathrm{B}$, com retirada de 20 canas por parcela, em 20 pontos distintos, escolhidos ao acaso mas dispersos por tôda a parcela, é pràticamente o mínimo possivel de material que nos pode dar uma precisão aceitável. A retirada de apenas duas canas por parcela, como fez VEIGA (1959), é, portanto, contra-indicado, pois daria, mesmo 
para o caso do método $B$, com nossos dados, um coeficiente de variação de cêrca de $8,5 \%$, que pràticamente impede a comprovação de qualquer diferença de composição da cana.

Já em ensaios de variedades as diferenças de composição podem ser bem maiores, de sorte que se poderá, em casos favoráveis, trabalhar com 10 a 20 canas por parcela.

\section{CONCLUSÕES}

1) $\mathrm{O}$ método $\mathrm{B}$, de colheita de certo número de canas por parcela, uma só de cada touceira e bem dispersas por tôda a área da parcela, é preferivel ao método $\mathrm{A}$, de coleta de canás próximas ou de uma mesma touceira.

2) No caso do teor de açúcar provável podem-se prever os coeficientes de variação do quadro seguinte, no caso de se usar o método $\mathrm{B}$, com $n$ canas por parcela.

\begin{tabular}{|c|c|}
\hline $\begin{array}{l}\text { N.o de canas por } \\
\text { parcela }(\mathrm{n})\end{array}$ & $\begin{array}{l}\text { Coeficiente de variação do açícar } \\
\text { provável por cento de cana no } \\
\text { caso do método B }\end{array}$ \\
\hline $\begin{array}{r}1 \\
2 \\
4 \\
10 \\
15 \\
20 \\
25 \\
30\end{array}$ & $\begin{array}{r}12,1 \% \\
8,5 \% \\
6,0 \% \\
3,8 \% \\
3,1 \% \\
2,7 \% \\
2,4 \% \\
2,2 \%\end{array}$ \\
\hline
\end{tabular}

3) É contra-indicado, em qualquer caso, usar amostras de menos de 10 canas por parcela.

4) Para casos de ensaios de adubação, com uma só variedade, em que há influência relativamente pequena sôbre a composição da cana, um mínimo de 20 colmos por parcela é aconselhável.

\section{SUMMARY}

The authors carried out 3 experiments on the sampling of sugar cane for technological determinations, one with each of the 
varieties Co 419, CB 40-69 and CB 41-58, in Piracicaba, State of São Paulo, Brasil. The main intent of the project was to compare 2 methods of sampling, namely:

1) Method A, where the sample is a hill (CATANI et al., 1959 ) or, more generally, 20 stalks all together in a randomly selected point of the furrow;

2) Method B, where 20 stalks are taken, from 20 points evenly spread out on the whole plot.

For the 3 varieties studied and for the data on Brix, pol, coefficient of purity, available sucrose and weight, analyses of variance were carried out. Further computations led to the following coefficients of variation.

Coefficients of variation for 20 stalk samples

\begin{tabular}{|c|c|c|c|}
\hline Variety & Characteristic & 20 stalks per hill & 1 stalk per hill \\
\hline CB $40-69$ & $\begin{array}{l}\text { Brix } \\
\text { Pol } \\
\text { Coefficient of purity } \\
\text { Available sucrose } \\
\text { Weight }\end{array}$ & $\begin{array}{l}4.8 \% \\
6.4 \% \\
2.1 \% \\
7.3 \% \\
6.6 \%\end{array}$ & $\begin{array}{l}1.9 \% \\
2.5 \% \\
0.83 \% \\
2.7 \% \\
6.9 \%\end{array}$ \\
\hline Co 419 & $\begin{array}{l}\text { Brix } \\
\text { Pol } \\
\text { Coefficient of purity } \\
\text { Available sucrose } \\
\text { Weight }\end{array}$ & $\begin{array}{r}5.3 \% \\
7.6 \% \\
2.9 \% \\
8.6 \% \\
21.2 \%\end{array}$ & $\begin{array}{l}1.8 \% \\
2.6 \% \\
1.0 \% \\
3.0 \% \\
6.5 \%\end{array}$ \\
\hline CB 41-58 & $\begin{array}{l}\text { Brix } \\
\text { Pol } \\
\text { Coefficient of purity } \\
\text { Available sucrose } \\
\text { Weight }\end{array}$ & $\begin{array}{r}2.8 \% \\
4.1 \% \\
1.8 \% \\
5.0 \% \\
10.9 \%\end{array}$ & $\begin{array}{l}1.4 \% \\
1.9 \% \\
0.8 \% \\
2.2 \% \\
6.2 \%\end{array}$ \\
\hline
\end{tabular}

For available sucrose, which is probably the most important characteristic studied, the average coefficient of variation for the 3 varieties was $2.7 \%$, for the case of method B, that is, 20 stalk samples, one stalk per hill. Assuming this coefficient of variation, in a trial with 5 treatments and 6 replications, in randomised blocks, the least significant difference among treatment means, at the $5 \%$ level, would be $4.7 \%$ of available sucrose by Tukey's test, and $3.3 \%$ by the $t$ test. For the case of method A the average coefficient of variation is $7.0 \%$ and, in similar conditions, the least significant difference would be $15.1 \%$ by Tukey's test, and $12.1 \%$ 
by the $t$ test. Since differences of available sucrose among treatments in experiments with fertilizers seldom are higher than 3 or $4 \%$ of the mean (PIMENTEL GOMES \& CARDOSO, 1958), method $B$ with a 20 stalk sample per plot gives more or less the minimum amount of cane to be sampled for technological determinations. In experiments with varieties, however, where differences may be assumed to be higher, a sample of 10 to 20 stalks one per hill, can be enough.

\section{BIBLIOGRAFIA}

ALMEIDA, J. R., O. VALSEHI, F. PIMENTEL GOMES, E. M. CARDOSO e N. CAMOLESI, 1952 - El Florescimento en la Variedad de Caña Co 421, Memoria de la Conf. An., As. Tecnol. Azuc. de Cuba, 100 pp., Havana.

ASSOCIATION OF OFFICIAL AND AGRICULTURAL CHEMISTS, 1960 - Official Methods of Analysis, 9.a ed., A. O. A. C., 418 pp., Washington.

BROWNE, C. A. e F. W. ZERBAN, 1941 - Physical and Chemical Methods of Sugar Analysis, 3.a ed.. John Wiley \& Sons, Nova York.

CATANI, R. A., H. C. ARRUDA, D. PELLEGRINO e H. BERGAMIN FILHO - 1959 - A absorção de Nitrogênio, Fósforo, Potássio, Cálcio, Magnésio, Enxofre e Silicio pela Cana-de-Açúcar, Co 419, e o seu Crescimento, em Função da Idade. Anais E. S. A. «Luiz de Queiroz»16: $167-190$.

COCHRAN, W. G. e G. M. COX - 1957 - Experimental Designs, 2.a ed., 611 pp. Nova York.

COURY, T., E. MALAVOLTA, F. PIMENTEL GOMES, J. D. P. ARZOLLA, M. O. C. BRASIL SOPRINHO, H. P. HAAG, F. A. F. MELO, R. F. NOVAES, G. RANZANI e L. MENARD - $1957-$ A Diagnose Foliar na Cana-rle-Acúcar, 28 pp., São Paulo.

PIMENTEI, GOMES, F. e ENO M. CARDOSO - 1958 - A Adubação da Cana-de-Açúcar, 116 pp., Piracicaba.

PIMENTEL GOMES, F. - 1963 - Curso de Estatística Experimental, 2.a ed., 384 pp., +15 tabelas. Piracicaba.

SPENCER, G. L. e G. P. MEADE - 1932 - Manual de Fabricantes de Azucar de Caña e Químicos Azucareros (trad.) 7.a ed., John Wiley \& Sons., 412 pp., Nova York.

VEIGA, F. MENEZES - 1959 - Estação Experimental de Campos Relatório do Instituto do Açúcar c do Alcool, 95 pp., Rio de Janeiro.

\section{AGRADECIMENTO}

Os autores agradecem à Refinadora Paulista S/A., que nos cedeu o material para o presente ensaio. Estes agradecimentos são extensivos ao Dr. Crucciano Crucciani, chefe da Secção Agrícola, da Usina Monte Alegre, em Piracicaba, e ao Sr. Antonio Brumharo, chefe do Setor Agrícola de Taquaral, da mesma usina. 